VoL. $61(2000)$ [405-413]

\title{
SMOOTHING THE DOMAIN OUT FOR POSITIVE SOLUTIONS
}

\author{
YAPING LIU
}

\begin{abstract}
For a given nonlinear partial differential equation defined on a bounded domain with irregular boundary, the available analytical tools are very limited in relation to the study of positive solutions. In this paper we first use weak convergence methods to show that for an elliptic equation of a certain type, classical positive solutions on nearby smooth domains approach a generalised positive solution on the given domain. The idea is then applied to sublinear elliptic problems to obtain existence and uniqueness results.
\end{abstract}

\section{INTRODUCTION}

In the modelling of many ecological problems using partial differential equations, the natural habitat of a certain species is usually formulated as a bounded domain in an $n$-dimensional Euclidean space. Since the pioneering work of Benoit Mandelbrot on fractals, a prevailing viewpoint is that a natural region has a highly irregular boundary. Theoretically, the presence of irregular points on the boundary makes it difficult to apply many of the analytical tools that are available. (Some definitions of regular boundary points can be found in [13] and [24].) For a given application problem, the usual practice is to take a domain with smooth or piece-wise smooth boundary, presuming that the solution behaviour of the problem in question should not display any dramatic change if the corners or edges of the domain are rounded off locally without affecting the domain shape on a large scale. But is this mathematically justified? This proves to be too general a question to have any definite answer.

In this paper we consider the following semilinear elliptic equation

$$
-\Delta u=f(x, u) \text { in } \Omega, \quad u=0 \text { on } \partial \Omega
$$

where $\Omega$ is a bounded domain in $R^{n}$ with boundary $\partial \Omega$. It is known that the solutions of (1) near an irregular point on the boundary can display singular behaviour, even if the problem is linear, that is, $f$ is independent of $u$. This is of course impossible if $\partial \Omega$ is smooth (with appropriate regularity assumption on $f$ ). On the other hand, we are going to show that as far as the existence of positive solutions of (1) is concerned, we can

Received 27th July, 1999

Copyright Clearance Centre, Inc. Serial-fee code: 0004-9727/00 \$A2.00+0.00. 
smooth the domain out to facilitate the mathematical analysis. More precisely, we first approximate $\Omega$ by a sequence of smooth domains $\left(\Omega_{n}\right)$. Then we use weak convergence methods in Lebesgue and Sobolev spaces to show that the sequence of classical solutions $\left(u_{n}\right)$ on the smooth domains $\left(\Omega_{n}\right)$ approaches a generalised solution $u$ on $\Omega$. It follows that if $u$ displays singular behaviour, then the classical solution $u_{n}$ on $\Omega_{n}$ becomes more and more singular-like as $n \rightarrow \infty$. When the methods are applied to a class of sublinear problems, a well known result is generalised to general bounded domains.

Domain perturbations have been studied in $[8,9,10,12]$ with certain restrictions on the domain $\Omega$. In our case the domain is arbitrary. We use monotone convergence to study the existence of positive solutions. For simplicity of presentation, we only consider the Laplace operator in equation (1). Most of the following results can be extended to general second order selfadjoint uniformly elliptic operators. In fact, the ideas used here clearly apply to a wider class of problems than elliptic equations.

\section{General Existence Results}

We first introduce the following definitions and notations.

1. A function $u \in W_{0}^{1,2}(\Omega) \cap L^{\infty}(\Omega)$ is a solution of (1) if $\int_{\Omega} \nabla u \nabla \phi$ $-f(x, u) \phi=0$ for all $\phi \in W_{0}^{1,2}(\Omega)$.

2. A solution $u$ is positive if $u>0$ almost everywhere in $\Omega$.

Since functions differing on a zero measure set are considered the same, a function $u$ is positive on $\Omega$ if $u(x)>0$ for all $x \in \Omega$.

3. A function $u \in W^{1,2}(\Omega) \cap L^{\infty}(\Omega)$ is a lower solution of (1) if $\int_{\Omega} \nabla u \nabla \phi$ $-f(x, u) \phi \leqslant 0$ for all $\phi \in W_{0}^{1,2}(\Omega)^{+}:=\left\{w \in W_{0}^{1,2}(\Omega): w \geqslant 0\right.$ on $\left.\Omega\right\}$ and $u \leqslant 0$ on $\partial \Omega$ in the sense that $\max \{u, 0\} \in W_{0}^{1,2}(\Omega)$.

An upper solution of $(1)$ is defined by reversing the above inequalities, while $u \geqslant 0$ on $\partial \Omega$ means $\min \{u, 0\} \in W_{0}^{1,2}(\Omega)$.

4. A sequence of domains $\left(\Omega_{n}\right)$ converges to $\Omega$ from inside if for any compact subset $K$ of $\Omega$ we have $K \subset \Omega_{n} \subseteq \Omega$ for all large $n$. Similarly, a sequence of domains $\left(\Omega_{n}\right)$ converges to $\Omega$ from outside if for any compact subset $K$ of $(\bar{\Omega})^{c}$ we have $K \subset \Omega_{n}^{c} \subseteq \Omega^{c}$ for all large $n$. Here $\Omega^{c}$ denotes the complement of $\Omega$ in $\boldsymbol{R}^{n}$.

It is easy to see that for any bounded domain $\Omega$ there exists a sequence of smooth domains $\left(\Omega_{n}\right)$ that converge to $\Omega$ from inside. In fact, the sequence $\left(\Omega_{n}\right)$ can be made increasing in the sense that $\Omega_{n} \subseteq \Omega_{n+1}$. In this case, $\Omega=\bigcup \Omega_{n}$. We can also choose the sequence $\left(\Omega_{n}\right)$ such that $\bar{\Omega}_{n} \subset \Omega$ for every $n$. Similar results hold for convergence of smooth domains from outside. 
5. Any function $u$ defined on a domain $\Omega$ is also viewed as a function on any $E \subset \Omega$ and on any $F \supset \Omega$, where $u$ on $E$ is defined by restriction and $u$ on $F$ is extended by zero, that is, $u=0$ on $F \backslash \Omega$.

6. $\lambda_{1}(\Omega)$ denotes the principal eigenvalue of the problem $-\Delta u=\lambda u$ in $\Omega$, $u=0$ on $\partial \Omega$.

Note that on any bounded domain the principal eigenvalue of a selfadjoint second order uniformly elliptic operator is defined. As the minimum value of the Rayleigh quotient of the operator, it is real, simple, and its corresponding eigenfunction can be chosen positive (see [13, Chapter 8]). Recently it was shown in [5] that similar results are true for non-selfadjoint elliptic operators.

Lemma 1. Let $\Omega$ be a bounded domain in $\boldsymbol{R}^{n}$, let $f$ be a Carathéodory function defined on $\bar{\Omega} \times \boldsymbol{R}$ such that for some $a(x) \in L^{2}(\Omega)$ and some $b>0,|f(x, t)|<a(x)+b|t|$ on $\Omega \times \boldsymbol{R}$, and let $\left(\Omega_{n}\right)$ be a sequence of smooth domains that converge to $\Omega$ from inside. If on each $\Omega_{n}$ equation (1) has a solution $u_{n}$ and the sequence $\left(u_{n}\right)$ is bounded in $L^{2}(\Omega)$, then every weak limit point of $\left(u_{n}\right)$ in $L^{2}(\Omega)$ is a solution of equation (1) on $\Omega$.

Proof: Because $u_{n} \in W_{0}^{1,2}\left(\Omega_{n}\right)$, the natural extension $u_{n}=0$ on $\Omega \backslash \Omega_{n}$ puts $u_{n} \in W_{0}^{1,2}(\Omega)$. Thus $\left(u_{n}\right)$ can be viewed as a sequence in either $W_{0}^{1,2}(\Omega)$ or $L^{2}(\Omega)$.

By the boundedness of $\left(u_{n}\right)$ and the weak compactness theorem, $\left(u_{n}\right)$ has a weak limit point in $L^{2}(\Omega)$. On the other hand, let $u$ be an arbitrary weak limit point of $\left(u_{n}\right)$ in $L^{2}(\Omega)$ and we show that $u$ is a solution of (1). By [2, Theorem 2.1], the superposition operator $F: L^{2}(\Omega) \rightarrow L^{2}(\Omega)$ defined by $F u(x)=f(x, u(x))$ maps bounded sets into bounded sets. Thus $f\left(\cdot, u_{n}(\cdot)\right)$ is a bounded sequence in $L^{2}(\Omega)$. Then by Hölder's inequality, the sequence $\int_{\Omega} f\left(x, u_{n}\right) u_{n}$ is bounded. Using the facts that $u_{n}=\nabla u_{n}=0$ on $\Omega \backslash \bar{\Omega}_{n}, m\left(\partial \Omega_{n}\right)=0$ because $\partial \Omega_{n}$ is smooth, $m$ denoting the Lebesgue measure in $\boldsymbol{R}^{n}$, and $u_{n}$ is a solution on $\Omega_{n}$, we have

$$
\int_{\Omega}\left|\nabla u_{n}\right|^{2}-f\left(x, u_{n}\right) u_{n}=\int_{\bar{\Omega}_{n}}\left|\nabla u_{n}\right|^{2}-f\left(x, u_{n}\right) u_{n}=\int_{\Omega_{n}}\left|\nabla u_{n}\right|^{2}-f\left(x, u_{n}\right) u_{n}=0 .
$$

This shows that $\left(u_{n}\right)$ is bounded in $W_{0}^{1,2}(\Omega)$. The compact imbedding $W_{0}^{1,2}(\Omega) \hookrightarrow L^{2}(\Omega)$ is valid for an arbitrary bounded domain $\Omega$ because of the zero boundary condition. Thus by the reflexivity of $W_{0}^{1,2}(\Omega),\left(u_{n}\right)$ has a subsequence, which we still denote by $\left(u_{n}\right)$, that converges to some $v \in W_{0}^{1,2}(\Omega)$ weakly in $W_{0}^{1,2}(\Omega)$ and strongly in $L^{2}(\Omega)$. It follows easily that $v=u$ in $L^{2}(\Omega)$. By the results in [2] again we see that the sequence $f\left(\cdot, u_{n}(\cdot)\right)$ converges strongly and thus weakly to $f(\cdot, u(\cdot))$ in $L^{2}(\Omega)$. Now for any fixed $\phi \in C_{0}^{\infty}(\Omega)$, we have supp $\phi \subset \Omega_{n}$ for all large $n$. Therefore

$$
\int_{\Omega} \nabla u \nabla \phi-f(x, u) \phi=\lim _{n \rightarrow \infty} \int_{\Omega} \nabla u_{n} \nabla \phi-f\left(x, u_{n}\right) \phi=\lim _{n \rightarrow \infty} \int_{\Omega_{n}} \nabla u_{n} \nabla \phi-f\left(x, u_{n}\right) \phi=0 .
$$

By the denseness of $C_{0}^{\infty}(\Omega)$ in $W_{0}^{1,2}(\Omega)$, we conclude that $u$ is a solution of (1) on $\Omega$. 
NOTE. If $\left(u_{n}\right)$ is uniformly bounded, then the conditions on $f$ in Lemma 1 can be fulfilled by the simple assumption $f \in C^{0}(\bar{\Omega} \times R)$. Clearly, this implies that $f$ is Carathéodory. We can modify $f(x, u)$ for $|u|$ large so that the conditions in Lemma 1 are satisfied.

Combined with the classical monotone method, the above lemma enables us to study the existence of positive solutions to a semilinear elliptic equation defined on an irregular domain by considering the problem on nearby smooth domains.

THEOREM 1. Let $E \subset F$ be bounded smooth domains and let $\Omega$ be any domain such that $E \subseteq \Omega \subseteq F$. Assume that $f(x, 0) \geqslant 0$ for $x \in \Omega$ and $f \in C^{\alpha}(\bar{F} \times[0, \infty))$ for some $0<\alpha<1$. If equation (1) has a lower solution $\underline{u}$ on $E$ and an upper solution $\bar{u}$ on $F$ with $0 \leqslant \underline{u} \leqslant \bar{u}$ and $0 \neq \underline{u} \in W^{2,2}(\Omega)$, then equation (1) has a positive solution $u$ on $\Omega$ with $\underline{u} \leqslant u \leqslant \bar{u}$. In addition, $u$ is the limit of a sequence of classical positive solutions on smooth domains converging to $\Omega$ from inside.

Proof: First we can define $f(x, u)$ for $u<0$ and redefine $f(x, u)$ for $u>\bar{u}$ so that Lemma 1 applies. Take any increasing sequence of smooth domains $\left(E_{n}\right)$ that converge to $\Omega$ from inside with $E_{1}=E$. It is easy to see that $\bar{u}$ is an upper solution of (1) on each $E_{n}$. By the monotone method, there is a classical solution $u_{1}$ of $(1)$ on $E_{1}$ with $\underline{u} \leqslant u_{1} \leqslant \bar{u}$. By the strong maximum principle, $u_{1}>0$ on $E_{1}$. Because $E_{1}$ has a smooth boundary, $u_{1}$ with natural extension is a lower solution on $E_{2}$ (see [4]). This implies the existence of a classical solution $u_{2}$ on $E_{2}$ with $u_{1} \leqslant u_{2} \leqslant \bar{u}$. By induction, we can find an increasing sequence $\left(u_{n}\right)$ with $u_{n} \leqslant \bar{u}$ so that each $u_{n}$ is a classical solution of (1) on $E_{n}$. It follows from the Monotone Convergence Theorem that the pointwise limit $u$ of $\left(u_{n}\right)$ is a strong limit of $\left(u_{n}\right)$ in $L^{2}(\Omega)$. Then Lemma 1 shows that $u$ is a positive solution of (1) on $\Omega$.

\section{Applications to Sublinear Problems}

In this section we apply the ideas used above to a class of sublinear elliptic equations. First we can easily extend $[19$, Lemma 2.1$]$ to its weak version.

LEMMA 2. Let $c(x) \in L^{\infty}(\Omega)$ and let $\mu_{1}(-\Delta+c)$ denote the principal eigenvalue of the Schrödinger operator $-\Delta+c$ on $\Omega$. Define a bilinear form $\mathcal{L}$ by $\mathcal{L}(u, v)$ $=\int_{\Omega} \nabla u \nabla v+c u v$ for $u, v \in W_{0}^{1,2}(\Omega)$.

(a) If there exists a function $u \in W_{0}^{1,2}(\Omega)$ such that $\mathcal{L}(u, u)<0$, then $\mu_{1}(-\Delta+c)<0$.

(b) If there exists a function $u \in W_{0}^{1,2}(\Omega)^{+}, u \neq 0$ such that $\mathcal{L}(u, v) \geqslant 0$ for all $v \in W_{0}^{1,2}(\Omega)^{+}$, then $\mu_{1}(-\Delta+c) \geqslant 0$.

ProOF:

(a) The result follows directly from the variational characterisation of the minimum eigenvalue. 
(b) Note that the condition here implies $(-\Delta+c) u \geqslant 0$ in the weak sense. An argument similar to that in [19] works.

To present the next theorem, we propose the following hypotheses on $f$ :

(H1) $f \in C^{\alpha}(\bar{\Omega} \times[0, \infty)), 0<\alpha<1$.

(H2) $\liminf _{t \rightarrow 0^{+}} f(x, t) / t>\lambda_{1}(\Omega)$ uniformly for $x \in \Omega$.

(H3) $\limsup _{t \rightarrow+\infty} f(x, t) / t<\lambda_{1}(\Omega)$ uniformly for $x \in \Omega$.

It is well known that if $\Omega$ has a smooth boundary, then hypotheses (H1)-(H3) imply the existence of a classical positive solution to equation (1). If in addition, $f(x, t) / t$ is strictly decreasing in $t$, then the positive solution is unique. This result has been developed by many authors in different forms and employed in many situations during the past few decades. See, for example, $[1,4,18,20,21,22]$. In particular, it is an indispensible tool for the decoupling technique in the study of elliptic systems (see $[6,7,15,21])$. On domains with irregular boundaries, we first prove the existence of positive solutions. For this purpose we replace (H1) and (H3) by the following technical condition:

(H3') There is a smooth bounded domain $F \supseteq \Omega$ such that $f \in C^{\alpha}(\bar{F} \times[0, \infty))$ and $\limsup _{t \rightarrow+\infty} f(x, t) / t<\lambda_{1}(F)$ uniformly for $x \in F$.

THEOREM 2. Let (H2) and $\left(\mathrm{H}^{\prime}\right)$ be satisfied. Then equation (1) has a positive solution.

PROOF: It is easy to see that the following one-sided continuous dependence of the principal eigenvalue on the domain holds: if a sequence of domains $\left(\Omega_{n}\right)$ converge to $\Omega$ from inside, then $\lambda_{1}\left(\Omega_{n}\right) \rightarrow \lambda_{1}(\Omega)$. Also well known is the fact that $\lambda_{1}(\Omega)$ decreasingly depends on the domain in the sense that $\Omega_{1} \subseteq \Omega_{2}$ implies $\lambda_{1}\left(\Omega_{1}\right) \geqslant \lambda_{1}\left(\Omega_{2}\right)$. Thus we can find a smooth subdomain $E \subset \Omega$ such that (H2) holds when $\Omega$ is replaced by $E$. It follows that there are arbitrarily small positive lower solutions on $E$ that can take the form $\varepsilon \phi(x)$ for a positive eigenfunction $\phi$ on $E$ and a small $\varepsilon>0$. (H3') implies that there are arbitrarily large upper solutions on $F$. In fact, an upper solution can be given by $K \psi(x)$, where $K$ is large positive number and $\psi$ satisfies $\psi(x)>0$ on $\bar{F},-\Delta \psi \leqslant \lambda \psi$ in $F$ for some $\lambda$ that is close to the principal eigenvalue of the problem $-\Delta u=\lambda u$ in $F, u=0$ on $\partial F$. Note that (H2) implies $f(x, 0) \geqslant 0$ for all $x \in \Omega$. Theorem 1 then implies that (1) has a positive solution on $\Omega$.

NotE. Consider the special case when $f(x, t)=f(t)$ is independent of $x$. (H3') is trivially satisfied if $\limsup _{t \rightarrow+\infty} f(t) / t \leqslant 0$. (This is the case for many logistic growth population models.) In general, $\left(\mathrm{H}^{\prime}\right)$ follows from ( $\mathrm{H} 3$ ) and the right continuous dependence of the principal eigenvalue on the domain in the sense that as $\left(\Omega_{n}\right)$ converges to $\Omega$ from outside, $\lambda_{1}\left(\Omega_{n}\right) \rightarrow \lambda_{1}(\Omega)$. One sufficient condition for such a continuous dependence is 
the "stability" of $W_{0}^{1,2}(\Omega)[3]$. This condition also makes it possible to approach positive solutions on $\Omega$ by classical positive solutions on smooth domains that converge to $\Omega$ from outside. Many other conditions are known that ensure the stability of $W_{0}^{1,2}(\Omega)$ (see, for example, $[\mathbf{2 3}, \mathbf{2 5}, \mathbf{2 6}])$.

Next we show the uniqueness of positive solutions. To do this, we first prove the existence of the smallest positive solution. This is again accomplished by using a sequence of classical positive solutions on smooth subdomains of $\Omega$.

THEOREM 3. Let (H1) and (H2) be satisfied.

(a) If equation (1) has positive solutions then it has a smallest positive solution.

(b) If $f(x, t) / t$ is strictly decreasing in $t$ for $(x, t) \in \Omega \times(0, \infty)$, then the positive solution is unique.

Proof: (a) Let $\mathcal{S}$ denote the collection of all positive solutions to (1). Assuming $\mathcal{S}$ is nonempty, we show that it contains a smallest element. Motivated by the method used in [11], we first apply Zorn's lemma to $\mathcal{S}$ to show the existence of minimal elements. Let $\left(u_{t}\right)_{t \in T}$ be a decreasing transfinite sequence in $\mathcal{S}$ and let $u=\inf \left\{u_{t}: t \in T\right\}$. Using the idea in the proof of $\left[14\right.$, Lemma 1.1] we can show that $\left(u_{t}\right)$ has a countable subsequence $\left(u_{n}\right)$ that converges strongly to $u$ in $L^{2}(\Omega)$. As in the proof of Lemma 1, this implies that $u$ is a nonnegative solution of (1). We claim that $u \neq 0$. Otherwise, $u_{n} \rightarrow 0$ in $L^{2}(\Omega)$. By (H2), there exists an $\varepsilon>0$ such that for small $t>0, f(x, t) / t>\lambda_{1}(\Omega)+\varepsilon$ for all $x \in \Omega$. Define $a_{n}(x)=g\left(x, u_{n}(x)\right) \wedge\left(\lambda_{1}(\Omega)+\varepsilon\right)$ where $g$ is the growth function $g(x, t)=f(x, t) / t$ for $t>0$ and $g(x, 0)=\liminf _{t \rightarrow 0^{+}} f(x, t) / t$. Then $f\left(x, u_{n}\right) \geqslant a_{n} u_{n}$. It follows from (H1), (H2) and the fact $u_{1} \in \stackrel{t \rightarrow L^{\infty}}{L^{\infty}}(\Omega)$ (by definition) that $a_{n} \in L^{\infty}(\Omega)$. Let $\theta$ be a positive eigenfunction corresponding to $\lambda_{1}(\Omega)$. It is easy to see that for all large $n, \int_{\Omega}|\nabla \theta|^{2}-a_{n} \theta^{2}<0$. By Lemma $2(\mathrm{a}), \mu_{1}\left(-\Delta-a_{n}\right)<0$. On the other hand, $\int_{\Omega} \nabla u_{n} \nabla \phi-a_{n} u_{n} \phi \geqslant \int_{\Omega} \nabla u_{n} \nabla \phi-f\left(x, u_{n}\right) \phi=0$ for all $\phi \in W_{0}^{1,2}(\Omega)^{+}$. Lemma 2(b) implies that $\mu_{1}\left(-\Delta-a_{\mathfrak{n}}\right) \geqslant 0$. This contradiction shows that $u$ is nontrivial. Because $u \in L^{\infty}(\Omega)$, we can choose $P>0$ large so that $(-\Delta+P) u=f(x, u)+P u \geqslant 0$. Thus $u$ is a positive solution by the strong maximum principle for weak solutions [13, Theorem 8.19]. It follows that $u$ is a lower bound of $\left(u_{t}\right)_{t \in T}$ in $\mathcal{S}$. So $\mathcal{S}$ has minimal elements.

Now let $u_{1}, u_{2}$ be two positive solutions to (1) and define $w=\min \left(u_{1}, u_{2}\right)$. Let $E$ be a smooth subdomain of $\Omega$ such that $\bar{E} \subset \Omega$ and such that (H2) is true with $\Omega$ replaced by $E . u_{1}, u_{2} \in L^{\infty}(\Omega)$ implies that $f\left(u_{1}\right), f\left(u_{2}\right) \in L^{\infty}(\Omega)$. By [13, Theorem 8.22], $u_{1}, u_{2} \in C^{\beta}(\bar{E})$ for some $\beta>0$. Thus $u_{1}, u_{2}>0$ on $\bar{E}$, which implies that $w>0$ on $\bar{E}$. It is clear that $u_{1}, u_{2}$ are upper solutions of (1) on $E$. So $w$ is also an upper solution on $E$ (see [11] or [27]). Hence there exists a classical positive solution $u_{0}$ on $E$ satisfying $u_{0} \leqslant w$. Applying this argument to an increasing sequence of smooth domains $\left(E_{n}\right)$ with $\bar{E}_{n} \subset \Omega=\bigcup E_{n}$, and using the idea in the proof of Theorem 1, we see that there is a positive solution $v$ on $\Omega$ with $v \leqslant w$. We conclude that (1) has a smallest 
positive solution.

(b) To show the uniqueness, let $u$ be an arbitrary positive solution of (1) and let $v$ be the smallest positive solution of (1). Then by the definition of solutions we have

$$
\int_{\Omega} \nabla u \nabla v-f(x, u) v=0=\int_{\Omega} \nabla v \nabla u-f(x, v) u .
$$

Thus $\int_{\Omega} f(x, u) v=\int_{\Omega} f(x, v) u$. This immediately implies $u=v$ by the fact that $f(x, t) / t$ is strictly decreasing in $t$ for $(x, t) \in \Omega \times(0, \infty)$.

As an easy application of the above results, we consider in the following a logistic equation defined on a 2-dimensional domain $A_{0}:=D_{R} \backslash\{(0,0)\}$, where $D_{R} \subset \boldsymbol{R}^{2}$ is the open disk with radius $R$ centred at the origin. Note that the boundary $\partial A_{0}$ contains an isolated irregular point $(0,0)$.

COROLlary 1. Let $a, b$ be real constants and let $b>0$. Then the equation

$$
-\Delta u=u(a-b u) \text { in } A_{0}, \quad u=0 \text { on } \partial A_{0}
$$

has a unique positive solution if and only if $a>\left(j_{0,1} / R\right)^{2}$, where $j_{0,1} \approx 2.4048$ is the smallest positive root of the first Bessel function $J_{0}(x)$.

Proof: Consider the annulus $A_{r}=\left\{(x, y): r^{2}<x^{2}+y^{2}<R^{2}\right\}$ for $0<r<R$. We have $A_{r} \subset A_{0} \subset D_{R}$. Thus $\lambda_{1}\left(A_{r}\right)>\lambda_{1}\left(A_{0}\right) \geqslant \lambda_{1}\left(D_{R}\right)$. Let $x_{0,1}$ be the smallest positive root of the equation $J_{0}(x) Y_{0}(x R / r)-Y_{0}(x) J_{0}(x R / r)=0$, where $Y_{0}(x)$ is the first Bessel function of the second kind. We have $\lambda_{1}\left(D_{R}\right)=\left(j_{0,1} / R\right)^{2}$ and $\lambda_{1}\left(A_{r}\right)=\left(x_{0,1} / r\right)^{2}$ (see [17]). As $r \rightarrow 0, A_{r} \rightarrow A_{0}$. It can be shown that $(R / r-1) x_{0,1} \rightarrow j_{0,1}$ (see [16]). Thus $x_{0,1} / r \rightarrow j_{0,1} / R$ and therefore $\lambda_{1}\left(A_{r}\right) \rightarrow \lambda_{1}\left(D_{R}\right)$. It follows that $\lambda_{1}\left(A_{0}\right)=\lambda_{1}\left(D_{R}\right)$. Now let $g(t)=a-b t$ be the growth function in the equation. We have $g(0)=a$ and $\lim _{t \rightarrow+\infty} g(t)=-\infty<\lambda_{1}\left(A_{0}\right)$. The existence of a unique positive solution then follows from Theorems 2 and 3 . Because $g$ is strictly decreasing in $t$, the condition $a>\lambda_{1}\left(A_{0}\right)$ is clearly also necessary.

\section{REFERENCES}

[1] H. Amann, 'Fixed point equations and nonlinear eigenvalue problems in ordered Banach spaces', SIAM Rev. 18 (1976), 620-709.

[2] J. Appell, 'The superposition operator in function spaces - a survey', Exposition Math. 6 (1988), 207-270.

[3] I. Babuška and R. Výborný, 'Continuous dependence of eigenvalues on the domain', Czechoslovak Math. J. 15 (1965), 169-178.

[4] H. Berestycki and P. L. Lions, 'Some applications of the method of super and subsolutions', in Bifurcation and Nonlinear Eigenvalue Problems, Lecture Notes in Mathematics 782 (Springer-Verlag, Berlin, Heidelberg, New York, 1980), pp. 16-41. 
[5] H. Berestycki, L. Nirenberg and S.R.S. Varadhan, 'The principal eigenvalue and maximum principle for second-order elliptic operators in general domains', Comm. Pure Appl. Math. 47 (1994), 47-92.

[6] J. Blat and K.J. Brown, 'Bifurcation of steady-state solutions in predator-prey and competition systems', Proc. Roy. Soc. Edinburgh Sect. A 97 (1984), 21-34.

[7] K.J. Brown, 'Spatially inhomogeneous steady-state solutions for systems of equations describing interacting populations', J. Math. Anal. Appl. 95 (1983), 251-264.

[8] E.N. Dancer, 'The effect of domain shape on the number of positive solutions of certain nonlinear equations', J. Differential Equations 74 (1988), 120-156.

[9] E.N. Dancer, 'The effect of domain shape on the number of positive solutions of certain nonlinear equations, II', J. Differential Equations 87 (1990), 316-339.

[10] E.N. Dancer and D. Daners, 'Domain perturbation for elliptic equations subject to Robin boundary conditions', J. Differential Equations 138 (1997), 86-132.

[11] E.N. Dancer and K. Schmitt, 'On positive solutions of semilinear elliptic equations', Proc. Amer. Math. Soc. 101 (1987), 445-452.

[12] D. Daners, 'Domain perturbation for linear and nonlinear parabolic equations', $J$. Differential Equations 129 (1996), 358-402.

[13] D. Gilbarg and N.S. Trudinger, Elliptic partial differential equations of second order, (2nd edition) (Springer-Verlag, Berlin, Heidelberg, New York, 1983).

[14] S. Heikkilä, 'On an elliptic boundary value problem with discontinuous nonlinearity', Appl. Anal. 37 (1990), 183-189.

[15] J. Hernández, 'Maximum principles and decoupling for positive solutions of reaction-diffusion systems', in Reaction-diffusion Equations, (K. J. Brown and A. A. Lacey, Editors), Oxford Science Publications, 1988 (Oxford University Press, New York, 1990), pp. 199-224.

[16] E. Jahnke, F. Emde and F. Lösch, Tables of higher functions, (6th edition) (McGraw-Hill, New York, 1960).

[17] J.R. Kuttler and V.G. Sigillito, 'Eigenvalues of the Laplacian in two dimensions', SIAM Rev. 26 (1984), 163-193.

[18] A. Leung, Systems of nonlinear partial differential equations: Applications to biology and engineering, Mathematics and its Applications (Kluwer Academic Publishers, Dordrecht, 1989).

[19] L. Li, 'Coexistence theorems of steady-states for predator-prey interacting systems', Trans. Amer. Math. Soc. 305 (1988), 143-166.

[20] L. Li and A. Ghoreishi, 'On positive solutions of general nonlinear elliptic symbiotic interacting systems', Appl. Anal. 14 (1991), 281-295.

[21] L. Li and Y. Liu, 'Spectral and nonlinear effects in certain elliptic systems of three variables', SIAM J. Math. Anal. 24 (1993), 480-498.

[22] P.L. Lions, 'On the existence of positive solutions of semilinear elliptic equations', SIAM Rev. 24 (1982), 441-467.

[23] J. Rauch and M. Taylor, 'Potential and scattering theory on wildly perturbed domains', J. Func. Anal. 18 (1975), 27-59.

[24] I.V. Skrypnik, Methods for analysis of nonlinear elliptic boundary value problems, Translations of Mathematical Monographs 139 (Amer. Math. Soc., Providence, RI, 1994). 
[25] F. Stummel, 'Perturbation theory for Sobolev spaces', Proc. Roy. Soc. Edinburgh, Sect. A 73 (1974), 5-49.

[26] F. Stummel, Perturbation of domains in elliptic boundary value problems, Lecture Notes in Mathematics 503 (Springer-Verlag, Berlin, Heidelberg, New York, 1975), pp. 110-135.

[27] G. Sweers, 'Semilinear elliptic problems on domains with corners', Comm. Partial Differential Equations 14 (1989), 1229-1247.

Department of Mathematics

Pittsburg State University

Pittsburg KS 66762-7500

United States of America 